Article

\title{
The Growth of Interest in Astronomical $X$-Ray Polarimetry
}

\author{
Frédéric Marin \\ Observatoire Astronomique de Strasbourg, Université de Strasbourg, CNRS, UMR 7550, 11 rue de l'Université, \\ F-67000 Strasbourg, France
}

Received: 15 January 2018; Accepted: 15 March 2018; Published: 19 March 2018

\begin{abstract}
Astronomical X-ray polarimetry was first explored in the end of the 1960s by pioneering rocket instruments. The craze arising from the first discoveries of stellar and supernova remnant $X$-ray polarization led to the addition of $X$-ray polarimeters to early satellites. Unfortunately, the inadequacy of the diffraction and scattering technologies required to measure polarization with respect to the constraints driven by $\mathrm{X}$-ray mirrors and detectors, coupled with long integration times, slowed down the field for almost 40 years. Thanks to the development of new, highly sensitive, compact $X$-ray polarimeters in the beginning of the 2000s, observing astronomical X-ray polarization has become feasible, and scientists are now ready to explore our high-energy sky thanks to modern $\mathrm{X}$-ray polarimeters. In the forthcoming years, several X-ray missions (rockets, balloons, and satellites) will create new observational opportunities. Interest in astronomical X-ray polarimetry field has thus been renewed, and this paper presents for the first time a quantitative assessment, all based on scientific literature, of the growth of this interest.
\end{abstract}

Keywords: X-rays; polarimetry; general; history of astronomy

\section{Introduction}

Since systematical studies of X-rays began in 1895 [1] and observational X-ray astronomy was born in the early 1960s [2], theoretical X-ray astronomy has predicted a wealth of results with polarimetry, but the conception of polarization-sensitive detectors took decades to achieve. It was only in the end of the 1960s that two Lithium-block Thomson-scattering polarimeters were attached to sounding rockets, targeting the brightest X-ray source known at that time: Sco X-1 [3]. More advanced experiments were conducted on Aerobee-350 rockets, loaded with two instruments in one payload: a Lithium scattering polarimeter and a network of 4 Bragg crystal polarimeters. This experiment led to the first detection of X-ray polarization from the Crab nebula [4], with the systematic effects being mitigated by rotating the detectors. Further efforts were put on rockets and satellites, such as Intercosmos-1, which measured the X-ray polarization from solar flares [5], and Ariel 5, equipped with a Bragg crystal spectrometer-polarimeter that produced an upper limit of $7.7 \%$ at three-sigma confidence for Sco X-1 [6]. The OSO-8 mission led to the sole positive detection of non-solar X-ray polarization, still in the Crab nebula $[7,8]$, with a linear polarization degree $\mathrm{P}=19.2 \pm 1.0 \%$ and a polarization position angle $\theta=156.4 \pm 1.4^{\circ}$ at $2.6 \mathrm{keV}$. A handful of upper limits were estimated for other objects, but most of them are unfortunately of marginal significance. The field of $X$-ray polarimetry was opened and several missions were envisioned to pursue the first discoveries. The Stellar X-ray Polarimeter (SXRP) was planned to fly on the Russian Spectrum-X Gamma Mission in the early 1990s [9] but the collapse of the Soviet Union precluded this satellite from taking flight. More dramatically, HEAO-2/Einstein revolutionized the way X-ray observations were made: a cosmic source became a cluster of detected photons in an imaging detector in the focus of optics. Rotation was no longer needed for spectroscopy or imaging and since then was considered a costly complication. The technical contrast 
between spectroscopy/imaging and polarimetry (which needed rotation), as well as the sensitivity mismatching between the different techniques, prevented the addition of complex, time-consuming, X-ray polarimeters in observatory missions. Even if rotation could be accomplished by rotating the detector on missions with telescopes (such as for SXRP), polarimeters were removed at different development stages (Einstein, AXAF) or from the very beginning (XMM, ATHENA) of many missions, as the integration times required to measure polarized fluxes were much longer than those for imaging or spectroscopy.

The recent breakthrough in X-ray polarization detectors was achieved in the beginning of the 2000s. The advent of electron-tracking polarimeters $[10,11]$ allowed for compact detectors capable of imaging processes together with polarization measurement. In comparison with old diffraction or scattering polarimeters, the increase in sensitivity is substantial: a factor of about hundred is expected with respect to the polarimeters on board the OSO-8. Coupled with state-of-the-art X-ray optics, the field of X-ray polarimetry is now ready to open again after 40 years of silence. The forthcoming launch of space-borne X-ray polarimeters [12] and the many balloon [13] and rocket [14] experiments that are currently planned clearly indicate a growing interest in X-ray polarimetry. This paper is intended to quantitatively show the enthusiasm of the research community for X-ray polarimetry. To do so, I will present in Section 2 the amount of registered publications on astronomical X-ray polarimetry, starting from the discovery of X-rays. I will then divide the database into subcategories to determine if all fields are growing at the same rate or if a specific domain is leading. Finally, I will conclude on the importance of $\mathrm{X}$-ray polarimetry in Section 3.

\section{Data Mining and Results}

To identify and collect all individual papers on astronomical X-ray polarimetry, I used the SAO/NASA Astrophysics Data System (ADS). It is a digital library portal for researchers in astronomy and physics, operated by the Smithsonian Astrophysical Observatory (SAO) under a NASA grant. There are more than 13.4 million records covering publications in astronomy, astrophysics, and general physics. A search by keywords was mandatory in order to narrow down the number of relevant papers. The list of keywords used for data mining comprised the terms "X-ray," "Rontgen," "polarization," "polarized," "polarimeter," "polarimetry," and "polarimetric," both in American English and British English. The resulting papers were examined individually to reject duplicates or false detections. The selected publications were stored in a database and classified per year and general subject. Six categories were created: "General," "Theory and instrumentation," "Solar, stellar and planets," "Strong gravity," "Strong magnetism," and "Galaxies (quiescent, clusters)." The "General" label stands for reviews, books, and historical notes that are not developing new aspects or mathematical equations in the field. The "Theory and instrumentation" label includes papers on the nature of X-rays and their fundamental properties. It also includes mechanical and technical papers about the development of instruments that goes along the aforementioned theoretical papers. All publications, regardless of the journal rank (A, B, etc.) were selected, together with lecture notes and proceedings. Only copies of seminar/conference presentations were rejected as they do not correspond to a publication sensu stricto. Eight hundred thirty-eight papers (as of the beginning of January 2018) were registered.

\subsection{Evolution of Publications on X-ray Polarimetry}

Figure 1 shows the results of the search and classification of papers relevant to the field of astronomical X-ray polarimetry. All subclasses of papers were merged, regardless of the scientific topic, in order to assess the growing interest of the community for high-energy polarimetry. The resulting histogram covers the period January 1895-January 2018. It immediately appears that the field stagnated for years between the discovery of $X$-rays and the launch of the first Lithium-block Thomson-scattering polarimeter, mounted inside an Aerobee- 150 rocket. This experiment led to a first upper limit on the polarization of Sco X-1 [3] and can be considered as the onset of the First Age of astronomical X-ray polarimetry. The cornerstones that dot the years between 1895 and 1968 (first rocket-borne experiment 
performed in July 1968, in search of X-ray polarization from Sco X-1 between 5.0 and $16.8 \mathrm{keV}$ ) are as follows: " $A$ " marks the discovery of X-rays. " $B$ " is when X-ray polarization is first mentioned [15]. " $\mathrm{C}$ " marks the publication of a fundamental paper summarizing the current understanding of the nature of gamma rays and X-rays [16]. " $\mathrm{D}$ " marks the flight of the aforementioned Aerobee-150 rocket, and one can see the impact of this experiment on the publication rate. The very next year, the publication number five times higher. Following celestial X-ray polarization measurement, the first measurements of polarization from non-thermal bremsstrahlung of solar flares were recorded in 1970 by the Intercosmos-1 mission (" $\mathrm{E}$ ") [5]. The number of publications kept increasing, up to 14 per year in 1972, which corresponds to the first non-solar astronomical X-ray polarization detection (" $\mathrm{F}$ ") [4]. As mentioned in the introduction, the target was the Crab Nebula, and the polarimeters were mounted on an Aerobee-350 rocket. Shortly after, the 8th Orbiting Solar Observatory (OSO-8) was launched in June 1975. While OSO-8's primary objective was to observe the Sun, four instruments were dedicated to observations of other celestial X-ray sources brighter than a few milliCrab. In particular, the Graphite Crystal X-ray Spectrometer on board allowed for X-ray polarization measurements in the 2-8 keV band (field of view: $3^{\circ}$ ). The analysis of 15 orbits of quick-look data on the Crab nebula showed that the polarization and position angles at 2.6 and $5.2 \mathrm{keV}$ were $15.7 \pm 1.5 \%$ at $161.1 \pm 2.8^{\circ}$ and $18.3 \pm 4.2 \%$ at $155.5 \pm 6.6^{\circ}$, respectively [7]. This is point " $G$ " on Figure 1, and it corresponds to the golden era of the First Age of X-ray polarimetry. The number of publication on the subject started to decrease and reached a plateau of $\sim 7$ publications per year in the 1980-2000 period. During this period of time, a number of significant but unfortunately unnoticed steps were taken. Of particular note, highlighted by point " $\mathrm{H}$," is the unfinished development of the Spectrum- $\mathrm{X}-\mathrm{Gamma}$ mission and its fully developed SXRP.

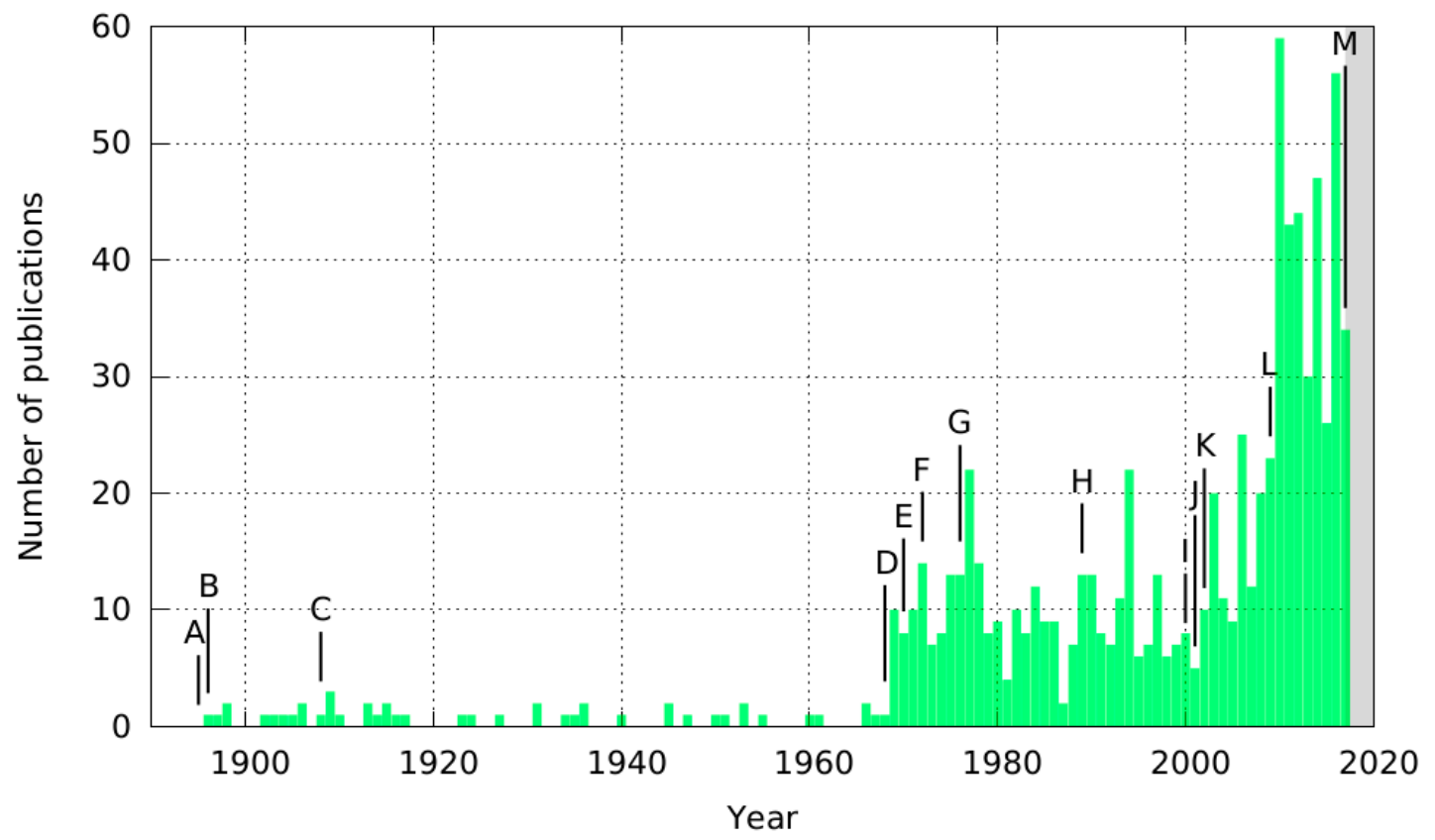

Figure 1. Number of publications focusing on astronomical X-ray polarimetry as a function of time. All types (refereed papers, proceedings, notes, etc.) and all scientific fields (solar physics, compact objects, etc.) are merged. Letters indicate cornerstones in the history of X-ray polarimetry and are detailed in the text.

When the design of the SXRP was in an advanced status, a modulation in the photocurrents of caesium iodide, gold, and aluminum photocathodes impinged at grazing angles by $60-200 \mathrm{eV}$ X-rays was found [17]. This modulation was much higher than what was predicted by usual transfer programs. Even though this signal was later found to be an artifact, it was the starter point for a node 
of scientists and engineers, who begun to reconsider photoelectric polarimetry as a potential solution to increase the sensitivity of X-ray polarimeters. Hence, the beginning of the new millennium saw several major innovations and attempts in measuring X-ray polarimetry. At Point " $\mathrm{I}$ ", $\mathrm{X}$-ray polarimetry experiments with balloon-borne gas proportional counters were conducted [18]. Detection of the X-ray polarization was only successful in a few sources, including Crab nebulae at 2.6 and $5.2 \mathrm{keV}$. Point "J" marks the development of the gas pixel detector, achieved in 2001 [10], which will carry out its first space flight in 2021. Point " $K$ " stands for both the development of the active-matrix pixel proportional counters [11] and the measurement of hard X-ray polarization of solar flares with RHESSI [19]. This marks the beginning of a Second Age for X-ray polarimetry. From this point (circa 2005), the amount of publications started to rise again at a rapid pace. From nine papers in 2005, the publication rate increased up to 59 in 2010. Since then, the averaged publication rate between 2010 and 2018 has been $\sim 2$ papers per year. This important growth is the result of the development of modern X-ray polarimeters and the beginning of recognition by the NASA, ESA, CNSA, JAXA, and other national space agencies that X-ray polarimetry represents a step forward in the study of our high-energy sky. In particular, the selection of the Gravity and Extreme Magnetism SMEX (GEMS) in 2009 was an important cornerstone (point "L" on Figure 1), but the mission was discontinued in May 2012. Point " $\mathrm{M}$ " is where we stand now: the Imaging X-ray Polarimetry Explorer (IXPE) mission has been planned [12] and will fly in 2021; several balloon and rocket experiments are envisioned, and numerous other small- or medium-sized spatial missions such as the enhanced X-ray Timing and Polarimetry mission (eXTP) are also planned [20].

\subsection{Publication per Field}

We saw that the amount of publications is growing at a significant rate. More importantly, half of the publication in astronomical X-ray polarimetry were written after 2005. With such a high infatuation, it is interesting to check whether the sub-categories are following the same trend.

We see in Figure 2 that a subcategory is clearly dominating the others between 1895 and 1968. The theoretical field was, as expected, predating any observation. Papers on specific celestial sources were almost inexistent, as a turning point in the field of high-energy astrophysics was the birth of X-ray astronomy with the first detection of a non-solar source [2]. Moreover, at that time, it was still unclear if X-rays could be polarized and if this polarization could be detected. However, with the onset of the First Age of X-ray polarimetry (circa 1970-1990) and the first flights of rockets and satellites mounted with polarimeters, papers on specifics subjects started to dominate the theoretical and instrumental fields. Led by observations of solar flares and the Crab nebula, the fields of "solar, stellar and planets" and "strong magnetism" science rose above the others. Numerical simulations also started with, e.g., the computation of the effect of special and general relativity onto the polarization of X-rays [21]. However, due to the lack of new observations, the interest of the community for X-ray polarization decreased in 1990. Only the field of "theory and instrumentation" kept growing, as, without new instruments or methods, it would be impossible to go on. Since the mid-1980s, this field presented an almost exponential rise, culminating far above the other subcategories. Only in the mid-2000s, after the development of the gas pixel detector and time projection chamber technologies, did the research community start to make efforts with $\mathrm{X}$-ray polarimetry again. Driven by the technological innovations, these fields of research started to publish new predictions on what would be observed. We note that the "solar, stellar and planets" and the "galaxies" scientific fields are lagging behind. For the former case, this is due to the absence of envisioned missions dedicated to solar and stellar $X$-ray polarimetry in the next decade. A new mission would certainly uplift the community to work on this field again. In the latter case, the very low $X$-ray fluxes, coupled with relatively low or inexistent polarization from galaxy clusters, naturally explains why the publication rate in this field is the lowest. Until the advent of extra-sensitive X-ray polarimeters, it is not mandatory to explore in greater detail the expected polarization of quiescent galaxies (save the Milky Way, where important and feasible observations await us [22-24]). 


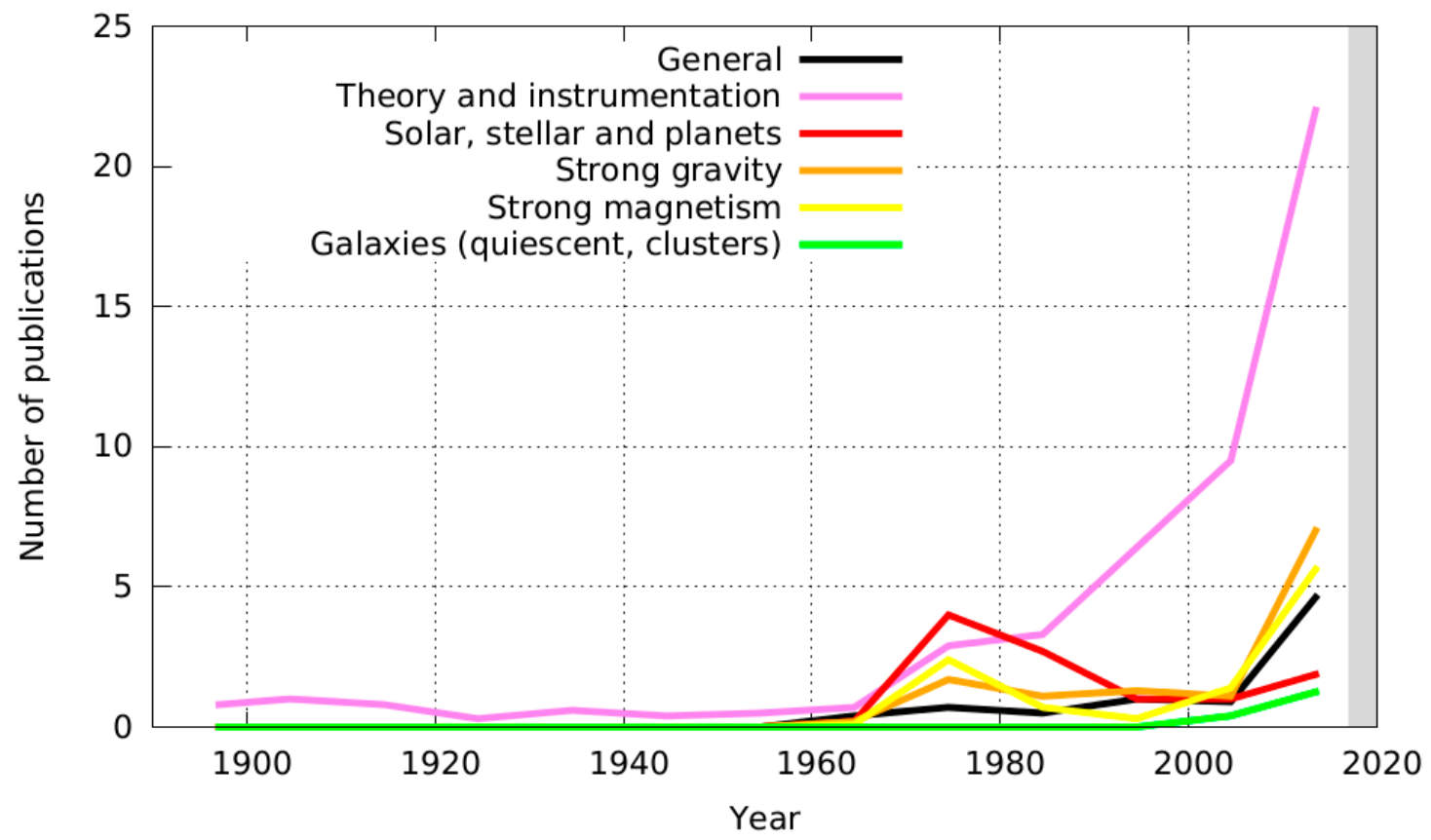

Figure 2. Number of publications focusing on astronomical X-ray polarimetry as a function of time. Several subcategories are highlighted: the black solid line represents general publications on X-ray polarimetry (reviews, historical notes); violet stands for publications concerning theory, instrumentation, and satellites, red for papers on solar, stellar, and exoplanet science; orange for papers on objects dominated by strong gravity effects (e.g., black holes); yellow for objects dominated by strong magnetic fields (e.g., neutron stars); the green solid line stands for papers related to galaxies (e.g., galaxy clusters or the Milky Way).

\section{Conclusions}

The number of publication related to astronomical X-ray polarimetry has been growing substantially since the beginning of 2000. Driven by new technologies and mature polarimeters, researchers in this field are publishing at an increasing rate, and the growth of interest for high-energy polarimetry is thus clearly measurable. The history of the field can be roughly summarized as follows: the First Age of (astronomical) X-ray polarimetry started in 1968 with the flight of the first Lithium-block Thomson-scattering polarimeter and ended circa 1980 with the termination of the OSO- 8 mission. At that time, despite the fact that polarimeters sharing the focal plane of $\mathrm{X}$-ray observatory missions suffered from the same difficulties that high-resolution spectroscopy did, the larger integration times needed by polarimeters to measure the polarized flux of cosmic sources and the mismatching between imaging/spectroscopic and polarimetric technologies were deemed too restrictive. This era was followed by a relatively quiescent period of 20 years, in which technical innovations were slow yet steady. The advent of new technologies in the beginning of the 2000s allowed for a renaissance in the field. This is the beginning of the Second Age for X-ray polarimetry. In comparison with the First Age, this era is showing slower development, as it takes much more time to send a polarimeter to high altitudes or into space than it did during the 1970s. However, this allows a stronger community of dedicated scientists to develop. The Second Age is not yet at its pinnacle. From cliodynamical (cliodynamics is a field of multidisciplinary research aimed at describing historical dynamics through mathematical models.) reasoning, one would expect the publication rate to keep growing as we approach observational results. The succession of envisioned missions, while will cover at least 15 years, should maintain a high enthusiasm in this scientific community, ensuring a long and remarkable Second Age for X-ray polarimetry. 
Acknowledgments: I am grateful to Martin C. Weisskopf and Enrico Costa who kindly agreed to share their historical knowledge on the subject with me, greatly improving the content of this paper. Additional information was kindly supplied by the two anonymous reviewers who participated to increase the quality of the manuscript. I would like to acknowledge the Centre National d'Etudes Spatiales (CNES) for providing funding to achieve this paper through the postdoctoral grant "Probing the geometry and physics of active galactic nuclei with ultraviolet and X-ray polarized radiative transfer". I would also like to acknowledge financial support from the University of Strasbourg, from the Astronomical Observatory of Strasbourg, from the Programme National Cosmologie et Galaxies (PNCG), and from the Programme National Hautes Energies (PNHE).

Conflicts of Interest: The authors declare no conflict of interest.

\section{References}

1. Röntgen, W.C. On a New Kind of Rays. Nature 1896, 53, 274-276.

2. Giacconi, R.; Gursky, H.; Paolini, F.R.; Rossi, B.B. Evidence for X Rays from Sources Outside the Solar System. Phys. Rev. Lett. 1962, 9, 439-443. [CrossRef]

3. Novick, R. X-Ray Polarization from SCO X-1 and Tau X-1. In Non-Solar X- and Gamma-Ray Astronomy; Proceedings from Symposium No. 37; Springer: Dordrecht, The Netherlands, 1970; Volume 37, p. 45.

4. Novick, R.; Weisskopf, M.C.; Berthelsdorf, R.; Linke, R.; Wolff, R.S. Detection of X-Ray Polarization of the Crab Nebula. Astrophys. J. 1972, 174, L1. [CrossRef]

5. Tindo, I.P.; Ivanov, V.D.; Mandel'Stam, S.L.; Shuryghin, A.I. On the Polarization of the Emission of X-Ray Solar Flares. Sol. Phys. 1970, 14, 204-207. [CrossRef]

6. Gowen, R.A.; Cooke, B.A.; Griffiths, R.E.; Ricketts, M.J. An upper limit to the linear X-ray polarization of SCO X-1. Mon. Not. R. Astron. Soc. 1977, 179, 303-310. [CrossRef]

7. Weisskopf, M.C.; Cohen, G.G.; Kestenbaum, H.L.; Long, K.S.; Novick, R.; Wolff, R.S. Measurement of the X-ray polarization of the Crab Nebula. Astrophys. J. 1976, 208, 125-128. [CrossRef]

8. Weisskopf, M.C.; Silver, E.H.; Kestenbaum, H.L.; Long, K.S.; Novick, R. A precision measurement of the X-ray polarization of the Crab Nebula without pulsar contamination. Astrophys. J. 1978, 220, 117-121. [CrossRef]

9. Kaaret, P.; Novick, R.; Martin, C.; Hamilton, T.; Sunyaev, R.; Lapshov, I.; Silver, E.; Weisskopf, M.; Elsner, R.; Chanan, G.; et al. SXRP. A focal plane stellar X-ray polarimeter for the SPECTRUM-X-Gamma mission. In Proceedings of the Conference on X-Ray/EUV Optics for Astronomy and Microscopy, San Diego, CA, USA, 7-11 August 1989; Volume 1160, pp. 587-597.

10. Costa, E.; Soffitta, P.; Bellazzini, R.; Brez, A.; Lumb, N.; Spandre, G. An efficient photoelectric X-ray polarimeter for the study of black holes and neutron stars. Nature 2001, 411, 662-665. [CrossRef] [PubMed]

11. Black, J.K.; Deines-Jones, P.; Ready, S.E.; Street, R.A. X-ray polarimetry with an active-matrix pixel proportional counter. Nucl. Instrum. Methods Phys. Res. Sect. A 2003, 513, 639-643. [CrossRef]

12. Weisskopf, M.C.; Bellazzini, R.; Costa, E.; Matt, G.; Marshall, H.L.; O’Dell, S.L.; Pavlov, G.G.; Ramsey, B.; Romani, R.W. Transforming Our Understanding of the X-ray Universe: The Imaging X-ray Polarimeter Explorer (IXPE). In Proceedings of the High Energy Astrophysics Division (HEAD) Divisional Meeting, Chicago, IL, USA, 17-21 August 2014; Volume 14, p. 116.

13. Chauvin, M.; Friis, M.; Jackson, M.; Kawano, T.; Kiss, M.; Mikhalev, V.; Ohashi, N.; Stana, T.; Takahashi, H.; Pearce, M. Calibration and performance studies of the balloon-borne hard X-ray polarimeter PoGO+. Nucl. Instrum. Methods Phys. Res. Sect. A 2017, 859, 125-133. [CrossRef]

14. Marshall, H.L.; Schulz, N.S.; Trowbridge Heine, S.N.; Heilmann, R.K.; Günther, H.M.; Egan, M.; Hellickson, T.; Schattenburg, M.; Chakrabarty, D.; Windt, D.L.; et al. The rocket experiment demonstration of a soft X-ray polarimeter (REDSoX Polarimeter). In Proceedings of the SPIE UV, X-Ray, and Gamma-Ray Space Instrumentation for Astronomy XX, San Diego, CA, USA, 6-8 August 2017; Volume 10397, p. 15.

15. Barkla, C.G. Polarisation in Röntgen Rays. Nature 1904, 69, 463. [CrossRef]

16. Bragg, W.H. The Nature of the $\gamma$ and X-Rays. Nature 1908, 78, 271. [CrossRef]

17. Fraser, G.W.; Pain, M.D.; Pearson, J.F.; Lees, J.E.; Binns, C.R.; Shaw, P.S.; Fleischman, J.R. Polarization sensitivity of X-ray photocathodes in the 60-200 eV band. In Proceedings of the International Society for Optical Engineering, San Diego, CA, USA, 22-23 July 1991; Volume 1548, pp. 132-148.

18. Hayashida, K.; Tsunemi, H.; Koike, T.; Horikawa, T.; Makino, F. X-ray polarimetry experiment with balloon borne gas proportional counters. Bull. Am. Astron. Soc. 2000, 32, 1265. 
19. McConnell, M.L.; Smith, D.M.; Emslie, A.G.; Hurford, G.J.; Lin, R.P.; Ryan, J.M. Measuring Hard X-Ray Polarization of Solar Flares with RHESSI. Bull. Am. Astron. Soc. 2003, 35, 616.

20. Zhang, S.; Feroci, M.; Santangelo, A.; Dong, Y.; Feng, H.; Lu, F.; Nandra, K.; Wang, Z.; Zhang, S.; Bozzo, E.; et al. eXTP: Enhanced X-ray Timing and Polarization mission. In Proceedings of the SPIE Astronomical Telescopes and Instrumentation, Edingurgh, UK, 26 June-1 July 2016; Volume 9905, p. 16.

21. Connors, P.A.; Stark, R.F. Observable gravitational effects on polarised radiation coming from near a black hole. Nature 1977, 267, 128-129. [CrossRef]

22. Churazov, E.; Sunyaev, R.; Sazonov, S. Polarization of X-ray emission from the Sgr B2 cloud. Mon. Not. R. Astron. Soc. 2002, 330, 817-820. [CrossRef]

23. Marin, F.; Karas, V.; Kunneriath, D.; Muleri, F. Prospects of 3D mapping of the Galactic Centre clouds with X-ray polarimetry. Mon. Not. R. Astron. Soc. 2014, 441, 3170-3176. [CrossRef]

24. Marin, F.; Muleri, F.; Soffitta, P.; Karas, V.; Kunneriath, D. Reflection nebulae in the Galactic center: Soft X-ray imaging polarimetry. Astron. Astrophys. 2015, 576, 19. [CrossRef]

(C) 2018 by the author. Licensee MDPI, Basel, Switzerland. This article is an open access article distributed under the terms and conditions of the Creative Commons Attribution (CC BY) license (http:/ / creativecommons.org/licenses/by/4.0/). 